\title{
Abstract Objects in a Metaphysical Perspective
}

\author{
Aleksandr Kulieshov ${ }^{1}$ \\ ${ }^{1}$ Cherkasy State Technological University \\ 460 Shevchenko Boulevard, 18006, Cherkasy, Ukraine
}

DOI: $10.22178 /$ pos.31-1

LCC Subject Category: BD95-131

Received 21.01.2018

Accepted 14.02.2018

Published online 25.02.2018

Corresponding Author:

klshv2014@gmail.com

(c) 2018 The Author. This article is licensed under a Creative Commons Attribution 4.0 License @)

\begin{abstract}
The article presents an unconventional although not absolutely unprecedented view on abstract objects defending the position of metaphysical realism. It is argued that abstract objects taken in purely ontological sense are the forms of objects. The forms possess some common characteristics of abstract objects, they can exist not in physical space and time and play a grounding role in their relation to concrete objects. It is stated that commonly discussed abstract objects - properties, kinds, mathematical objects - are forms.
\end{abstract}

Keywords: metaphysics; abstract objects; metaphysical realism; nominalism; forms; spatio-temporal characteristics; grounding.

\section{INTRODUCTION}

Abstract objects are of two major interests for philosophers and scientists. Firstly they are the referents of scientific and everyday speech. Without referring to abstract objects scientific and everyday language would be deprived of the means of generalization what would be fatal for science and nearly so for everyday communication. Secondly they are the key item of metaphysics. Their clear understanding reveals what sort of reality we live in. The problem of abstract objects is commonly admitted as central to modern philosophy dealing with metaphysical problems.

Philosophical texts of the last decades show special and not decreasing interest for the problem which has the form of the discussion between two conceptions of abstract objects. One is traditionally marked as metaphysical realism another as nominalism. Sometimes realism is associated with Platonism, sometimes there is more sophisticated distinction between realism and Platonism like that argued in the book of W. L. Craig [3, p.13]. The difference is in the scope of abstract objects but both variants accept some. So we may call the both "metaphysical realists". The controversy of metaphysical realists and nominalists has long history which cannot be traced here. Still some recent traits should be noted. There is generally accepted standard understanding of its subject matter. The defining characteristics of abstract objects are three according to S. Cowling: 1) lack of any causal role in the world; 2) lack of spatiotemporal location; 3) special epistemic access to them [2, p.2]. This is quite standard representation of abstract objects. Philosophers discern different kinds of them, particularly: mathematical entities, properties and, propositions [2, p. 3]. One may add natural kinds as one of the most significant group of abstract objects.

The theme of abstract objects is well elaborated. New ideas in this domain of philosophy seem inevitably to be a reminder of very old and well known ones. Such is the J. Porter Moreland's interpretation of the problem of universals as that concerning "One and Many" problem [12, p. 11]. There is also thoroughly developed standard systems of arguments and counter-arguments for and against realism and nominalism. Arguments against nominalism in details are formulated in a paper of Z. G. Szabo [14], most widely used among them - indispensability argument and the context principle [14, pp. 29, 33]. One should note also the refutation of nominalism by G. Rosen [13]. Well-grounded arguments against realism may be found in C. Dorr's chapter in "Contemporary Debates in Metaphysics" which are based on the contrast between superficial 
and more profound way of talking about abstract objects [4, p. 9-11]. F. MacBride analysing the contemporary debate on universals finds the essence of differences in the understanding by philosophers of the degree and character of orderliness in the Universe [11, p. 278].

The contemporary realists-nominalists dispute reminds of a grandmasters' chess game in which all the debut moves are long known, counted and foreseen. Arguments and counter-arguments become more and more subtle but do not go beyond some limits. The dispute seems to move by circle. The necessity is felt to view the problem from another point which may be not absolutely new but has not been in a focus of interest at the recent phase of debate. The objective of this paper is to present such unconventional view defending the position of metaphysical realism. Two main questions will be answered: How else (besides known definitions) can be defined the objects generally called abstract? What does their real existence mean?

\section{RESULT AND DISCUSSION}

We have to define with new degree of precision the objects of our interest. It is the task to find new boundaries of a known phenomenon. First of all let`s take apart two principally incompatible viewpoints on abstract objects. One is ontological, another - epistemological. They are commonly go together at philosophical texts of analytic orientation. Abstract objects are defined having properties of lacking locality, causal neutrality and special cognitive access as though these properties are of the same type. It seems natural to regard abstract objects as a result of abstraction. Logic of such research strategy is that abstractness is a sort of mental representation, which may well refer on not refer to reality. The central point of discussion becomes the problem of reference to abstract objects. How imperceptible objects could be referred to is qualified as the fundamental point in philosophical theories of reference [8, p. 50]. The search for truth-conditions based on the context principle of G. Frege becomes in this case the way of explanation [8, p.51]. The aim is, according to M. Dummett thought, to find "some content" while identifying the referent of abstract singular terms [5, p. 65]. Why reality is liable to such constructive efforts by human mind is out of question here. The most suitable image of this treatment of the problem is, in M. Eklund words, when "reality considered in itself is like some amorphous dough and our concepts are like cookie cutters, carving up reality into objects" [6, p. 383].

Another vision of reality (represented in this paper) presupposes that reality is not chaotic. Such vision needs the differentiation of reality from the human cognitive process. Accordingly there are two principally different ways of being for abstract objects - as they exist by themselves (if they exist at all) and as they exist within human cognition. Existence of abstract objects regardless of their cognition is seen as a special aspect of their problem. The question is whether this aspect can be differentiated from the cognitive one. The common belief of analytical philosophers (especially nominalists) is that it cannot. The decisive premise of this paper, on the contrary, is that independently existing abstract objects are conceivable. Our cognitive ability of abstraction may go further than constructing abstract objects. We can abstract as well from the process of abstraction leaving 'within the brackets" just the objects and their objective existence. This is purely metaphysical point of view and from now on abstract objects will be understood this way only.

Limiting and purifying the understanding of abstract objects we need to part with equivocation of existing objects and objects' existence. Existence is not a rare ground to the solution of the abstract objects problem. Contrast between abstract and concrete objects is often seen as the two types of existence. It is not a real solution but the transference of the difficulties in abstract objects analyses to the new and more obscure sphere of existence. The perspective of finding right decision becomes more vague as G. Imaguire reasonably notices "we have far too many different conceptions of existence; and possibly worse, we have different concepts of existence" [9, p. 376]. There is another way to solve the problem of abstract objects by means of existence analyses. One does not identify abstract objects with a special form of existence but uses the known, well studied forms. Such is the juxtaposition of concrete and abstract objects with actual and possible existence. This conception unites the spheres of abstract objects and possible worlds [1, p. 238]. As a matter of fact this conception ruins the very phenomenon of abstract objects as they become indiscernible from the possible objects. Our analysis is based on recognizing the actual existence of abstract objects 
as only this way the problem becomes worth thinking about. Another kind of using the known form of existence in explaining abstract objects is the presentist conception of A. Filomeno. Abstract entities exist according to it in the same way as past and future things exist [7, p. 177]. At least the former and he latter share such properties as the lack of locality and causal inactiveness. This analogy is interesting and may have some ontological grounds but hardly reveals the essence of abstract objects. Abstract objects seem to be something more fundamental than a mere representation of a form of time.

The main idea of this part of discussion is that existence of abstract objects is not abstract existence. It is no use to differentiate existence because of its extreme abstract character. Existence is existence and our intuitive understanding of the term is enough here. Surely the existence of abstract objects as real is extremely important to explain. Such explanation appears to be the ultimate point of discussion. Still the very term of existence does nothing to solve the problem. We may part with it to continue the search.

Standard set of abstract objects properties includes lack of causal power. Note that negative property is already questionable. What do abstract objects possess instead of causal power? Lack of interaction with concrete spatial objects makes it difficult to define the place of abstract objects in the world structure. It looks like there is no connection between different kinds of objects. Lack of causal power should not be just stated, it must be explained and the ties of abstract objects with other parts of reality must be revealed. Not going into discussion of causal problems in metaphysics let's just take into account far from clear understanding of this phenomenon. There are too many explanations of the essence of causal relation and how it works in modern philosophy.

When it is said that abstract objects lack causal power the cause is understood as a physical interaction factor. Causality means the contact between physical objects in time and space leading to some spatial changes. Abstract objects obviously don't act this way. But if abstract objects exist they have to be somehow involved in causal processes, though apparently indirectly. They have to be connected with concrete causes and effects. This connection may be not directly causal but it must have something in common with causality. So we have to view the abstract- concrete relation in a broader context. The most general context is marked by the term 'determinism', causality in particular is a kind of determinism. If we cannot say about abstract objects in terms of causality, we can analyse the deterministic role of abstract objects.

The sort of determinism associated with abstract objects is grounding. Abstract objects (if they exist) ground the existence of concrete objects. By grounding we mean the objects existence of which is necessary for the existence of other (grounded) objects but not vice versa. Without abstract objects (as we conceive them) no concrete objects exist. It may be said however that without concrete objects abstract ones don't exist as well and so this is not the case of grounding. The answer is this. When it goes about particular objects a concrete object does not exist without abstract but any abstract object can exist without any of the concrete. Surely abstract objects need for its existence something concrete. But all concrete objects do not pre-exist before every one of them. And every one of them is not unique and therefore is grounded by abstract objects.

It is commonly acknowledged that abstract objects are not located in space. Less common are statements about their timelessness. It is mostly this point which causes difficulties in defending the reality of abstract objects. Absolute spaceless and timeless existence is the mark of strictly Platonist type of metaphysical realism. Today the main line is represented by a more moderate kind of realism. Abstract objects are not absolutely excluded from time and space. Not possessing "a determinate temporal and spatial properties of the same kind as concrete physical objects or events", as S. Klausen points out, they "should not commit us to view them as transtemporal or trans-spatial" [10, p. 4]. Even if abstract objects are not themselves spatiotemporal in physical sense they somehow are connected with spatiotemporal objects. They exist by means of spatiotemporal objects, so they are indirectly spatiotemporal themselves. But obviously this locality of abstract objects is indirect. They lack immediate physical locality. How it is possible looks somewhat like a mystery, if we are not inclined to use the supernatural world conception.

It may be that this is the principal point in resolving the problem of abstract objects. We are in need to change our physical outlook according to claims of metaphysics and this is not encouraged in our today's naturalistic discourse. One possible 
way to go out of trouble is not to refuse from the space-time concept but to generalize it. We may present locality in physical sense as not the only or even the necessary characteristic of space and time taken in a universal perspective. Then we could speak about abstract space and time drawing the line between the abstract and the concrete analogously to objects. For mathematically thinking minds at least it seems not unnatural.

The problem of deterministic relation of physically non-spatial abstract and physical spatial objects looks even more complex. Any ties in physical world are seen by the modern theoretical mind as fundamentally spatial. Even admitting non-spatial objects and their physical effectiveness we need to answer the question: what reality connects both abstract and physical objects spatial or non-spatial? One instance of such questionable relation is that of mind and body. Abstract objects in general are no less problematic in this sense. And again we have to construct some sort of common environment for physically spatial and physically non-spatial objects if we intend to provide a place for abstract objects within the known to us Universe.

For closer defining abstract objects in metaphysical sense we have to make this notion purely ontological. It is the task to abstract from abstraction, concentrating exclusively on object. We should search for something within objects (taking the word "within" in metaphorical sense not having in mind spatial location). It should be a part of object in most general sense of the term "part" (not spatial) and something coinciding in the same time with an object. There is a wellknown term for such an item, namely "the form ".

Taking general idea from Aristotle we should try to define it more precisely. The form as it seems at first sight can be identified as structural parts of an object connected in some way. For instance geometrical form is made of parts connected by some spatial relation. Let's take more strict definition - a form is a complex of an object's constituents being in one type relation. This relation makes the whole out of parts of an object and differentiates one whole from other such wholes. The form apparently is not identical with its constituents, it is something more complex. It is also not a mere sum of constituents, it embrace their relation in its peculiarity (not relation itself but specific relation). Neither it is a mere relation, it is something being in relation. An object obviously has not one form but many as there are many relations in which parts of an object may be connected. It is acceptable to say also about one general form of an object compiled of other forms.

Naturally forms are not everything in physical object (although an abstract or - better to say - a formal object is identical with its forms). Talking about something non-formal in physical objects it would be pertinent to apply one more Aristotelian term - "matter". Matter is, first of all, the uniqueness of an object, something which cannot be duplicated therefore not existing in different locations. Forms coincide in a way with objects and it may raise the question about their existence. If a form (say - a general form) is identical with an object we have no reason to admit it as something specially existing. Metaphysical understanding of existence however makes this situation unproblematic. "Existence of something" in the most general and abstract sense means just "differing of something from everything other than this". To exist is to be different from all other entities. Forms differ from everything which is not a form particularly from formalized objects. Therefore forms do exist.

Locality and non-locality (in physical sense) is based on different types of relations. There are spatial relations which are created by spatial parts of objects and tie objects to special (unique) localities. There are, conversely, non-spatial relations - structural, functional, typological, which in themselves are realized without localization in space. Relations, as it was stated before, define the character of forms. Hence forms may be local and non-local. One can assume as well that every physical object has both spatial and non-spatial forms. Spatial forms define localization of objects. It may be called "the local forms" of an object. A local form is made of material parts of an object and in this sense may be termed also "the material form". There are, besides, non-local forms of an object. They are made of parts which relate independently of locality of a physical object. Components of such forms may have locality but their locality don't influence the form, locality is left behind non-local forms. Their peculiarities are not defined by locality, so they exist out of physical space in some sense. Non-local forms lacking spatial features have another type of identification. They are not identical by identical placement. Their identity therefore is qualitative (not numerical). Nothing differentiates one form from another if they are partially or completely identical in qualities. So one form (or its part) 
may be represented in locally different physical objects.

Still it is the question how non-local forms are represented (or "instantiated") in local objects. It seems that we may use material forms as an intermediary between non-local forms and local (physical) objects. Another problem is with a type and direction of dependence between forms and material objects. The consideration concerning necessary presence of forms in formalized material (particularly physical) objects should be answered. Forms at least of physical objects do not exist apart from these objects. But the contrary is also true. Physical objects do not exist without any forms. There is no use to discuss what is the primary reality in causal or temporal sense. It is obvious that there is some dependence from both sides. It is also discernible that in the opposite directions dependence is different. Forms are dependent on material objects as structurally incomplete reality on structurally complete reality. Material objects are dependent on forms as something grounded on its grounds.

There are various types of abstract objects: among them properties, natural kinds, mathematical objects. Are those entities forms according to how the forms were defined in this paper? Properties, according to P. van Inwagen, are "ways in which a thing can be like a thing" [15, p. 121]. It looks plausible but may be rewritten in the terms of this paper like "relations in which parts of a thing are connected". Properties from this point of view are forms taken in their aspect of relations connecting their components. Strictly speaking properties are not forms but are formal entities, they belong to forms.

Natural kinds look like much closer entities to our definition of forms. Kinds' characteristics include both relations and components of the objects of one kind. Every such unity forms a part of an individual entity common with other individuals. As this common part lacks spatial characteristics it appears to be one and the same in different objects. So it may be said that individual objects carry in themselves the form of their kind.

Mathematical objects are often discussed as the most representative abstract objects. The major interest is shown for numbers. Do the numbers fit our definition of forms? As numbers are coun- terparts of properties in some sense it seems correct to provide them with similar formal role. Numbers represent that aspect of forms which consists in quantitative relations of components of a (complex) object (a set for instance). The belonging of geometrical forms to the domain of forms is intuitively evident. So, mathematical objects are a type of forms together with properties and kinds.

One more type of entities formal in their essence worth noting. These are mental representations: thoughts, images, perceptions, memory, consciousness etc. The may be more or less abstract but they are apparently formal. Mental representations fall within the definition of forms because they connect in non-local (physically) and nonmaterial way components of outer objects. Nonmaterial and non-local components of outer objects and their relations become immaterial representations but fully preserve their formal aspect. Mental phenomena may be described as non-local forms differentiated in some mysterious way from local ones and from material objects.

\section{CONCLUSION}

The problem of abstract objects existence, characteristics, and functions is the main problem of metaphysics. Metaphysical view presupposes the reduction of the discussion solely to the ontological aspect of the problem. Taken in purely ontological sense abstract objects are the forms of objects. The forms exist, being the necessary component of every real entity and at the same time not merging into form-bound entities, existing specifically. Differing from objects forms may be local and non-local, may exist in physical space or not in physical space. Non-local forms are represented in local forms which are in its turn represented in material objects. One non-local form may be represented in different material objects and in different locations. The forms are the ground of material objects but they depend on the latter as structurally incomplete reality. Commonly discussed abstract objects - properties, kinds, mathematical objects - are forms. The most interesting type of the forms is mental representations as this is the only known to scientific reason entities of purely formal character. 


\section{REFERENCES}

1. Cowling, S. (2013). The Way of Actuality. Australasian Journal of Philosophy, 92(2), 231-247. doi: 10.1080/00048402.2013.815239

2. Cowling, S. (2017). Abstract Entities. London; New York: Routledge, Taylor \& Francis Group.

3. Craig, W. L. (2017). God and Abstract Objects: The Coherence of Theism: Aseity. Cham: Springer.

4. Dorr, C. (2008). There are no abstract objects. In T. Sider, J. Hawthorne, \& D. W. Zimmerman (Eds.), Contemporary Debates in Metaphysics (pp. 32-64). Oxford: Blackwell.

5. Duke, G. (2013). Dummett and the problem of abstract objects. Teorema, 32(1), 61-75.

6. Eklund, M. (2008). The picture of reality as an amorphous lump. In T. Sider, J. Hawthorne, \& D. W. Zimmerman (Eds.), Contemporary Debates in Metaphysics (pp. 382-396). Oxford: Blackwell.

7. Filomeno, A. (2016). Abstract Entities in a Presentist World. Metaphysica, 17(2). doi: 10.1515/mp2016-0014

8. Heck, R. (2011). Frege's Theorem. Oxford: Clarendon Press.

9. Imaguire, G. (2015). The Platonism vs. Nominalism Debate from a Meta-metaphysical Perspective. Revista Portuguesa de Filosofia, 71(2-3), 375-398. doi: 10.17990/rpf/2015_71_2_0375

10. Klausen, S. H. (2013). Approaching the abstract: Building blocks for an epistemology of abstract objects. Semiotica, 2013(194). doi: 10.1515/sem-2013-0019

11. MacBride, F. (2009). Universals: the contemporary debate. In R. Le Poidevin (Ed.), The Routledge Companion to Metaphysics (pp. 276-285). London: Routledge.

12. Moreland, J. P. (2014). Universals. Abingdon: Routledge.

13. Rosen, G. (1993). The refutation of nominalism. Philosophy of Logic, 21(2), 149-186.

14. Szabó, Z. G. (2005). Nominalism. In M. J. Loux, \& D. Zimmerman (Eds.), The Oxford Handbook of Metaphysics (pp. 11-45). Oxford: Oxford University Press.

15. van Inwagen, P. (2004). A Theory of Properties. In D. W. Zimmerman (Ed.), Oxford Studies in Metaphysics. Vol. 1 (pp. 107-138). Oxford: Oxford University Press. 